\title{
ACTION SUR LES GERMES DE MAMMITE DU SUPERPHOSPHATE DE CHAUX EN SOLUTION AQUEUSE ET DANS LA LITIËRE
}

\author{
par
}

\author{
B. SEVEL et M. PLOMMET
}

Station Centrale de Mierobiologie et Recherches laitières, Jouy-en-Josas

L'épandage du superphosphate de chaux sur la litière des étables et des bergeries n'était, à l'origine, qu'une méthode d'amélioration de la composition des fumiers [1] ; elle est devenue depuis quelques années, en France; une méthode "à la mode» de lutte contre certaines maladies du bétail, les mammites et le piétin en particulier [2].

Aucune preuve sérieuse de l'efficacité de la méthode contre les mammites n'a été apportée jusqu'ici [3]. On mentionne des pourcentages de mammites cliniques pendant une période de temps donnée, sans tenir compte ni des infections latentes, ni de la nature des germes responsables, ni d'aucun des innombrables facteurs connus, susceptibles d'influencer l'apparition des mammites. Les essais que nous avons pu suivre de notre côté se sont soldés par des échecs. Cependant, nous avons pensé que la vogue dont jouit la méthode actuellement méritait de retenir l'attention et que, derrière des observations présentées volontairement comme empiriques et un peu mystérieuses, se cachait peut-être une solution pleine d'intérêt au problème des mammites.

A côté des hypothèses plutôt fantaisistes avancées pour expliquer l'action du superphosphate épandu sur la litière (modification du microbisme de l'étable, influence de l'ammoniac de l'air sur la nutrition azotée des animaux...) l'hy pothèse de Goret et Pilet [4] a retenu notre attention. Ayant consta té que le superphosphate de chaux en solution aqueuse a une activité bactériostatique et bactéricide remarquable, ces auteurs [5] pensent "qu'une telle méthode de désinfection (l'épandage sur la litière) pourrait constituer une arme intéressante dans la prophylaxie de certaines maladies infectieuses $)$.

Les germes des mammites ne se reproduisent pas dans le milieu extérieur; ils y survivent seulement; le pouvoir bactériostatique du superphosphate n'a donc pas d'intérêt dans ce domaine.

Par contre, l'action bactéricide peut présenter un intérêt : il est possible que la désinfection de la litière réduise sensiblement la contamination bien que la transmission de l'infection se fasse moins par l'intermédiaire de la litière que par celui des manchons de la machine à traire ou des mains du trayeur. Dans ce travail, nous avons étudié l'action bactéricide du superphosphate de chaux sur quelques germes de mammites, en solution aqueuse, d'une part, 
sur la litière de bergerie et la peau de la mamelle de la brebis, d'autre part.

\section{Première Partie}

\section{ACTION BACTÉRICIDE DU SUPERPHOSPHATE DE CHAUX ET DE SES CONSTITUANTS EN SOLUTION AQUEUSE}

Goret et Pilet ont montré qu'une solution filtrée de superphosphate contenant $15 \mathrm{gr} .7$ par litre de phosphore, résultant de la dissolution de 200 grammes de superphosphate dans 1 litre d'eau, a une action bactéricide totale après un temps de contact allant de 15 minutes à 5 heures, selon les germes étudiés; ils estiment que l'acidité de la solution, dont le $p H$ est de 3 , n'est pas seule en cause dans cette action bactéricide, mais que, probablement, les ions en solution participent à cette action.

L'action bactéricide d'un antiseptique en solution aqueuse varie avec le $p \mathrm{H}$ de.cette solution; aussi, la mesure du pouvoir bactéricide n'a de signification qu'à un $p H$ donné. Nous avons étudié l'action bacténicide de quelques constituants de la solution de superphosphate à différents $p H$. Pour mesurer eette action, nous nous sommes placés dans des conditions telles qu'une différence, même faible, de pouvoir bactéricide soit perceptible : la mesure du nombre de survivants après un temps de contact donné avec des solutions diluées nous a paru la méthode la plus fructueuse.

\section{I. - Matériel}

1. Solutions de superphosphate; solutions des constituants.

Le superphosphate résultant de l'action de l'acide sulfurique sur le phosphate tricalcique naturel a une composition légèrement variable selon l'origine des minerais. Il est constitué essentiellement de sulfate de calcium et de phosphate monocalcique, en proportion sensiblement équivalente. Les phosphates bi et tricalcique, l'acide phosphorique, l'acide sulfurique, s'y trouvent en petite quantité, ainsi que les métaux suivants ( teneur moyenne pour cent) [6] :

$\begin{array}{llll}\mathrm{Fe}_{2} 0_{3}: 1,6 & & \mathrm{Cr}_{2} 0_{3}: 0,10 \\ \mathrm{Al}_{2} 0_{3}: 1,0 & & \mathrm{CuO}: 0,014 \\ \mathrm{~V}_{2} 0_{5}: 0,06 & & \mathrm{MnO}_{2}: 0,006 \\ \mathrm{Mg} 0 & : 0,5 & & \mathrm{Zn0}: 0,8 \\ \mathrm{Ti}_{2}: 0,08 & & \mathrm{Ag} \quad: \text { traces }\end{array}$

Le superphosphate est partiellement soluble dans l'eau. La fraction soluble est constituée surtout de phosphate monocalcique et d'un peu de sulfate de calcium; la fraction insoluble, essentielle- 
ment de sulfate de calcium. Les solutions de superphosphate à concentration égale ou supérieure à $0,5 \%(\mathrm{p} / \mathrm{v})$, ont un $p \mathrm{H}$ de $3-3,2$. Le $p \mathrm{H}$ des solutions des sels de calcium de l'acide phosphorique est stable aux environs de $3-3,2$ (diacides) et de $6,8-7,0$ (monoacides) ; nous avons choisi ces $p \mathrm{H}$ pour mesurer l'action bactéricide du superphosphate et de ses constituants.

Au cours de nos essais, nous avons utilisé des solutions de superphosphate à $1 \%$ et à $0,5 \%(\mathrm{p} / \mathrm{v})$. Dans ces solutions, la fraction monocalcique a une concentration sensiblement moitié moindre $(0,5 \%$ et $0,25 \%)$, soit une molarité de 0,02 et $0,01 \mathrm{M}$.

Pour cette raison, nous avons comparé l'action bactéricide du superphosphate à $1 \%$ ou $0,5 \%$ à celle des phosphates mono et bicalciques à molarité 0,02 ou $0,01 \mathrm{M}$, selon le cas. A ces concentrations, le phosphate monocalcique est soluble mais le phosphate bicalciqu est partiellement insoluble. Pour éviter les erreurs qui pourraient résulter des différences de concentration en ions $\mathrm{PO}_{4}$, en raison d'une insolubilité partielle, les essais ont été faits parallèlement avec des solutions de phosphates mono et bipotassique de même molarité.

Le $p \mathrm{H}$ des solutions acides a été ajusté à $3-3,2$ par $\mathrm{H}_{2} \mathrm{SO}_{4}$ ou $\mathrm{H}_{3} \mathrm{PO}_{4}$. Le $p \mathrm{H}$ des solutions neutres et de la solution témoin de superphosphate a été ajusté à 6,8-7,0 par $\mathrm{NaOH}$ ou $\mathrm{NH}_{4} \mathrm{OH}$. Aux solutions de phosphates $0,01 \mathrm{M}$, on a ajouté séparément: 1) sulfate de calcium à saturation. 2) les ions Zinc et Chrome sous forme de $\mathrm{ZnSO}_{4}$ et $\mathrm{CrO}_{4} \mathrm{~K}_{2}$ à la concentration à laquelle ils se trouvent dans la solution de superphosphate correspondante $(0,5 \%)$.

Les solutions ont été utilisées sans stérilisation ou après stérilisation par tyndallisation à $55^{\circ}$; dans ce dernier cas, le $p \mathrm{H}$ a été vérifié après stérilisatíon. Dans les essais avec Streptococcus agalactiae, les solutions de superphosphate ont été faites dans l'eau physiologique à $0,85 \%$ de CINa.

\section{Souches microbiennes.}

Les germes de mammite les plus fréquemment rencontrés actuellement sont les staphylocoques (vaches et brebis), divers streptocoques - le plus important d'entre eux étant le streptocoque de la mammite contagieuse de la vache - et, plus rarement, les colibacilles. Trois souches de staphylocoques (n ${ }^{\text {os }} 80-33,80-38,80-40$ ) de mammites de brebis ont été utilisées dans tous nos essais; une souche de Str. agalactiae (no 47-1) et une souche de Escherichia Coli (no 130-29) ont été utilisées avec le superphosphate seul. Toutes les souches présentent les caractères typiques de l'espèce à laquelle elles appartiennent. Elles étaient toutes isolées récemment de mammites aiguës. 


\section{II. - Méthode}

Les suspensions de corps microbiens dans l'eau distillée ou dans l'eau physiologique (Str. agalactiae) sont préparées à partir de culture de 15 heures en bouillon ordinaire ou en bouillon tamponné glucosé (Str. agalactiae). Le titre de ces suspensions est établi par numération sur milieux gélosés tamponnés apprès dilution convenable en eau distillée (ou physiologique) tamponnée à $p \mathrm{H} 7$ par 3 grammes d'un mélange de phosphates mono et bipotassiques pour 1 litre.

Aussitôt après cette numération de base une même quantité de la suspension ( $1 \mathrm{ml}$.) est répartie dans les différentes solutions dont on veut tester le pouvoir bactéricide. Après des durées de contact de 15-30 minutes, 1 heure, 2 heures, 3 heures (ou 24 heures) à la température du laboratoire, on dénombre les germes survivants par numération en milieu gélosé. L'utilisation d'un liquide de dilution et d'un milieu de culture fortement tamponnés permet de limiter exactement le temps d'action des sels bactéricides agissant en milieu acide.

\section{III. - Résultats et Discussion}

Les graphiques $1,2,3,4$ et 5 expriment quelques-uns des résultats obtenus. Le nombre de germes est porté en ordonnée, le temps de contact en abscisse.

Le pouvoir bactéricide de chaque solution est ainsi représenté par la pente de la courbe correspondante : à une pente nulle correspond un pouvoir bactéricide nul, à une pente maximum, un pouvoir bactéricide élevé.

L'examen des courbes montre que le superphosphate en solution à $p H 3$ a une action bactéricide particulièrement nette sur les streptocoques et les staphylocoques, mais beaucoup moins nette sur les colibacilles. Après neutralisation par la soude ou par l'ammoniaque les solutions de superphosphate n'ont pratiquement plus aucune action bactéricide. On observe quelques petites divergences selon les souches.

Les phosphates monocalcique et monopotassique en solution à $p \mathrm{H} \mathrm{3-3,2}$ ont une action bactéricide moins rapide que celle du superphosphate. Les résultats sont d'ailleurs légèrement variables d'une expérience à l'autre avec le phosphate monocalcique; il est probable que la solubilité mal définie de ce produit est à l'origine des divergences observées.

L'adjonction des ions Chrome ou Zine aux solutions acides de phosphate monopotassique permet de reproduire l'action bactéricide du superphosphate. Le sulfate de calcium, par contre, n'a aucune action. A $p H$ 6,8-7,0, les solutions de phosphates de calcium 


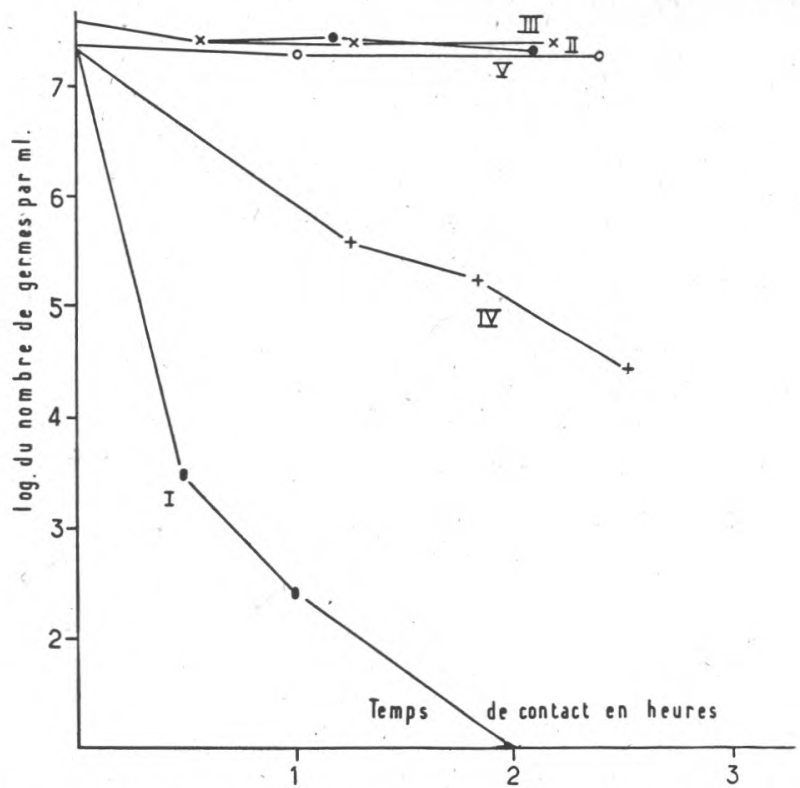

Fig. 1. - Action bactéricide du superphosphate de chaux en solution aqueuse. A. Staphylocoque (n ${ }^{\circ} 80-40$ ). I, superphosphate à $1 \%$ et à $p H 3$ II, superphosphate à $1 \%$ et à $p H 7$ (par $\mathrm{NaOH})$. III, superphosphate à $1 \%$ et à $p \mathrm{H} 7$ (par $\left.\mathrm{NH}_{4} \mathrm{OH}\right)$.

B. Colibacille (no 130-29). IV, superphosphate à $0,5 \%$ et à $p H$ 3. V, superphosphate à $0,5 \%$ et à $p \mathrm{H} 7$ (par $\mathrm{NaOH})$.

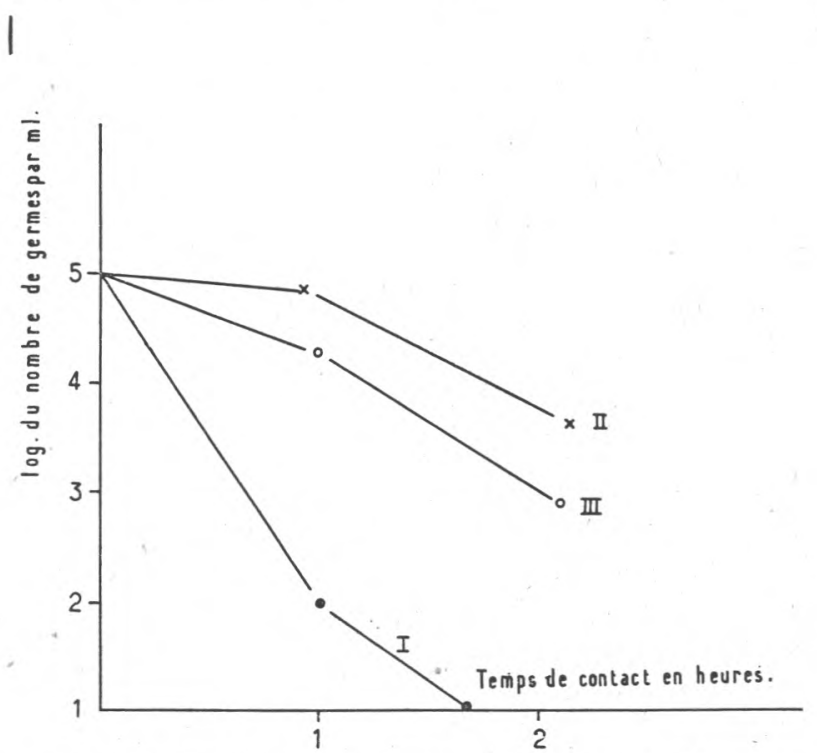

Fij. 2. - Action bactéridide du superphosphate de chaux en solution aqueuse. Streptococcus aqalactiae (n $\left.{ }^{0} 47-1\right)$ I. superphosphate à $0,5 \%$, dans l'eau physiologique à $8,5 \%$ o et à $p$ H 3. II. superphosphate à $0,5 \%$ dans l'eau physiologique à $8,5 \%$ ot à $p \mathrm{H} 7$ (par $\mathrm{NaOH}$ ). III. Eau physiologique à $8,5 \%$. 


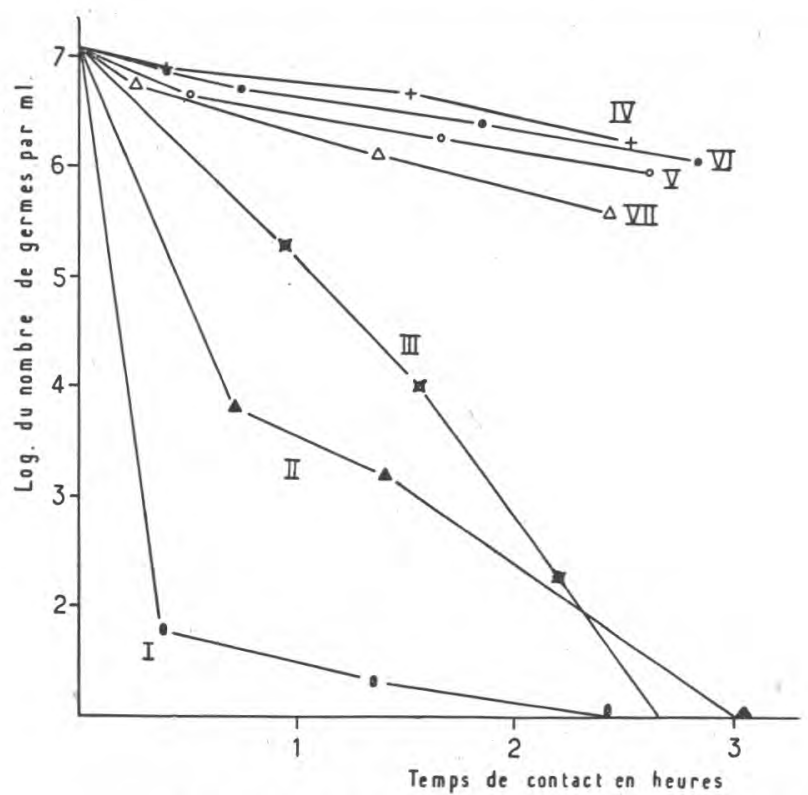

Fig. 3. - Actions bactéricides comparées du superphosphate et de différentes solutions de phosphates. Staphylocoque (no 80-38).

A. Solutions à $p H$ 3-3,2. I. superphosphate à $0,5 \%$. II. Phosphate monocalcique 0,01 M-acide sulfurique. III. Phosphate monopotassique $0,01 \mathrm{M}$ acide sulfurique.

B. Solutions à $p H$ 6,8-7. IV. superphosphate à $0,5 \%$. V. Phosphate bicalcique à $0,01 \mathrm{M}$. VI. Mélange de phosphates mono et bipotassique $0,01 \mathrm{M}$. VII. Eau distillée.

et de potassium additionnées ou non des ions Chrome ou Zinc n'ont aucune action bactéricide.

Ainsi, l'action bactéricide du superphosphate est le résultat de l'action propre du phosphate monocalcique (ions $\mathrm{PO}_{4}^{---}$) et des ions métalliques en solution parmi lesquels les ions $\mathrm{Cr}^{+++}$et $\mathrm{Zn}^{++}$ sont probablement les plus importants. Ces actions ne se manifestent qu'en milieu acide. Goret et Pilet ont émis l'hypothèse que « l'action bactéricide du superphosphate en solution aqueuse est due à l'action propre des ions $\mathrm{PO}_{4}^{---}$, à l'acidité $\mathrm{du}$ milieu et probablement aux ions métalliques en solution ". Nos résultats confirment cette hypothèse en ce qui concerne les ions $\mathrm{PO}_{4}^{---}$et les ions métalliques; mais ils diffèrent en ce sens que l'acidité du milieu est le facteur primordial pour que se manifeste l'action des uns et des autres.

Dans ces conditions, on peut se demander quelle peut être l'action du superphosphate épandu sur la litière des étables et bergeries quand on sait que ces litières, normalement alcalines en raison de la 


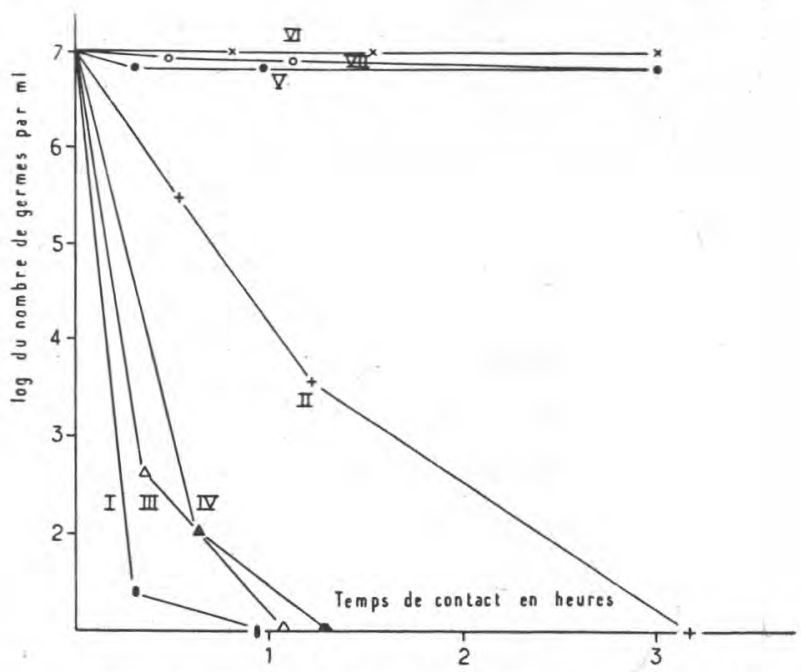

Fig. 4. - Actions bactéricides comparées du superphosphate et de certains de ses constituants. Staphyloco que no 80-40.

A. Solutions à $p \mathrm{H} 3-3,2$ I. superphosphate à $0,5 \%$. II. Phosphate monopotassique 0,01 M-acide sulfurique. III. Phosphate monopotassique 0,01 M, acide sulfurique - sulfate de zinc. IV. Phosphate monopotassique 0,01 M acide sulfurique - chromate de potassium.

B. Solutions à $p H$ 7. V. mélange de phosphates mono et bipotassique 0,01 M. VI. mélange de phosphates mono et bipotassique 0,01 M-sulfate de zinc. VII. mélange de phosphates mono et bipotassique 0,01 M-chromate de potassium.

production d'ammoniac, sont simplement ramenées au voisinage de la neutralité par l'adjonction de ce sel acide.

\section{Deuxième partie}

\section{ACTION BACTÉRICIDE DU SUPERPHOSPHATE DE CHAUX SUR LA LITIÈRE DE BERGERIE ET SUR LA PEAU DE LA MAMELLE DES BREBIS}

Les essais in vitro laissent à penser que, dans la litière, le superphosphate perd toute activité bactéricide du simple fait de sa neutralisation. Cependant, les différences très grandes qui existent entre le tube à essais contenant une solution díluée et la litière, où les conditions de concentration et de durée de contact ne sont pas comparables, ne permettent pas une simple transposition des résultats. De plus l'observation des animaux (brebis, truie) placés dans des loges recevant du superphosphate montre que ceux-ci se " poudrent " littéralement la peau de l'abdomen et des flancs en se couchant sur la litière. 


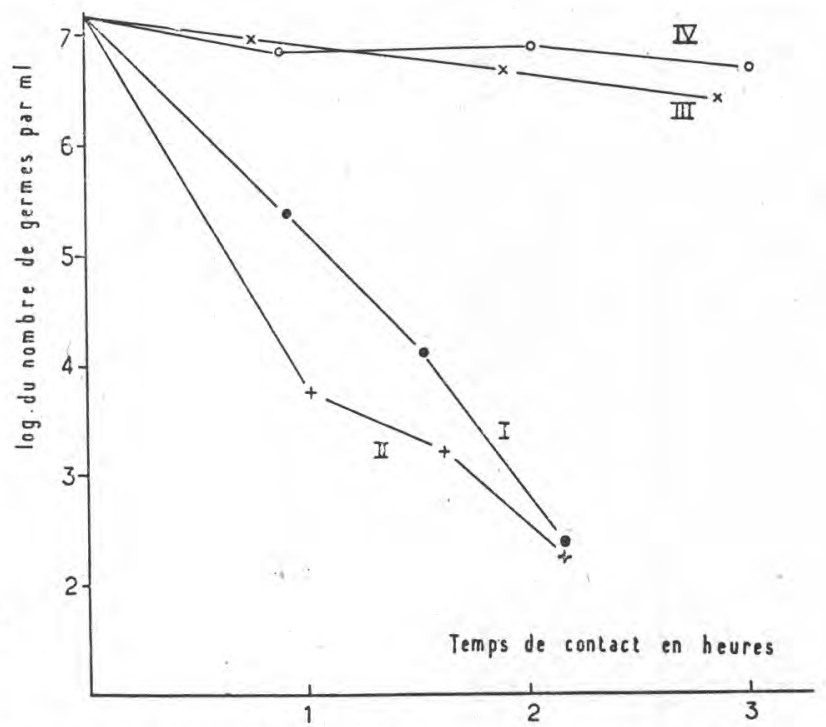

Fig. 5. - Action bactéricide du phosphate de potassium, saturé ou non de sulfate de calcium.

Staphylocoque n० 80-38.

A. Solutions à $p H$ 3-3,2. I. phosphate monopotassique $0,01 \mathrm{M}$-acide sulfurique. II. phosphate monopotassique 0,01 M-acide sulfurique-sulfate de calcium.

B. Solutions à $p H$ 7. III. mélange de phosphates mono et bipotassique 0,01 M. IV. mélange de phosphates mono et bipotassique 0,01 M-sulfate de calcium.

On peut imaginer que le superphosphate réalise de la sorte une désinfection de la peau, dont on sait le rôle très important dans la conservation et la transmission des germes de mammites.

C'est pourquoi nous avons, dans cette deuxième partie, recherché si l'adjonction de superphosphate à la litière de bergerie modifiait la survie des staphylocoques et des streptocoques dans la litière (méthode directe et méthode des porte-germes) et sur la peau de la mamelle des brebis (méthode de l'écouvillon).

\section{I. - Matériel}

1. Locaux. Nombre d'animaux.

Deux cases non contiguës d'une bergerie expérimentale du C.N.R.Z. (Brouessy) ont été utilisées. Dans les cases, d'une superficie d'environ 8 mètres carrés chacune, cinq brebis ont été logées pendant toute la durée des essais pour "faire" et entretenir une litière naturelle. 


\section{Litière.}

Le premier jour, les deux cases reçoivent de la paille fraîche en quantité suffisante pour constituer une litière épaisse et propre. Après constitution de celle-ci, on ajoute seulement la quantité de paille nécessaire pour l'entretenir. L'une des deux cases a reçu le premier jour 4 kilogrammes de superphosphate de chaux, puis, tous les jours suivants, 125 grammes par mètre carré (soit 200 grammes par bête).

Dans les conditions décrites ci-dessus, la litière recevant le superphosphate est d'apparence beaucoup plus sèche; de ce fait, elle n'exige pour être entretenue dans de bonnes conditions de propreté qu'une plus petite quantité de paille.

3. Souches microbiennes. Préparation des suspensions.

a) Staphylocoques.

Dans les essais préliminaires, les trois souches utilisées avaient le même comportement. Nous n'avons retenu par la suite que la souche 80-38 déjà citée da ns la première partie de ce travail.

La souche est cultivée en bouillon ordinaire à $37^{\circ}$ pendant 15 heures. Dans la méthode directe, le bouillon est utilisé tel quel. Dans la méthode des porte-germes, les corps microbiens sont repris dans de l'eau distillée tamponnée à $p H$ 7 (solution à $0,1 \%$ d'un mélange de phosphates mono et bipotassique).

b) Streptocoques.

Deux streptocoques ont été utilisés dans la méthode des portegermes : la souche $n^{0} 47-1$ de Str. agalactiae et la souche $n^{0} 10-38$ de Str. uberis. Cette dernière, isolée récemment d'un cas de mammite chronique de vache, possède le caractère Camp positif; on peut ainsi la rechercher sur milieu TKT. La culture est faite en bouillon glucosé tamponné et la suspension en eau physiologique tamponnée à $0,1 \%$.

4. Milieux.

a) Germes totaux.

La numération des germes totaux a été faite sur gélose nutritive ordinaire.

b) Staphylocoques.

La recherche des staphylocoques coagulase positive à partir de produits aussi contaminés que le fumier est délicate. Elle suppose l'emploi de milieux sélectifs sur lesquels les caractères morphologiques des colonies permettent de distinguer les staphylocoques coagulase positive des autres staphylocoques, microcoques et autres 
bactéries non inhibées. Sur le milieu de Chapman classique, rien ne permet de distinguer les staphylocoques pathogènes des innombrables microcoques fermentant la mannite, contenus dans le fumier. Sur 200 colonies testées, considérées comme typiques sur Chapman, aucune n'avait les caractères habituels (hémolyse) des souches pathogènes. Les milieux de Ludlam [7] et de Ludlam modifié par Chapman [8] sont, par contre, beaucoup trop inhibiteurs pour les quatre souches que nous avons essayées (une souche $\alpha \beta$ isolée de mammite de brebis, deux souches $\beta$ de mammite de vache, une souche $\alpha$ humaine). Le milieu de Zebovitz, Evans et Niven [9], dont nous avons ramené, après de nombreux essais, les concentrations respectives de glycocolle de 1 à $0,5 \%$ et de tellurite de potassium de $0,02 \%$ à $0,0075 \%$, donne par contre, d'excellents résultats à condition d'ajouter le tellurite de potassium en solution non stérilisée au moment de couler le milieu en boîtes : sur ce milieu, les staphylocoques pathogènes sont les seuls à donner, après 36 heures à $37^{\circ}$, de larges colonies noires (caractère vérifié sur 200 souches). Les autres germes non inhibés ont, soit des colonies de taille moyenne et grises (staphylocoques et microcoques non pathogènes), soit des colonies petites et très noires (streptocoques fécaux et pédiocoques), soit des colonies géantes gris-nois, avee un liséré périphérique transparent (pseudomonas). Le nombre de colonies retrouvées sur ce milieu par rapport à la gélose au sang est de 1 pour 3 (Staphylocoque 80-38).

c) Streptocoques.

La mise en évidence et le dénombrement de Str. agalactiae et de Str. uberis a été faite sur milieu TKT, après 18 heures d'incubation à $37^{\circ}[10]$.

\section{II. - Méthodes}

\section{A. - Recherche dans la litière}

\section{Méthode directe.}

Une surface délimitée des cases en expérience reçoit, par arrosage superficiel, des corps microbiens en suspension dans le bouillon de culture. Dans chaque zône, un double prélèvement de 50 grammes de litière est fait immédiatement, puis après 24 et 48 heures. Le premier est introduit dans un bocal de 500 millilitres d'eau distillée stérile tamponnée à $p \mathrm{H} 7(0,3 \%$ de phosphate), placé sur shaker et agité 10 minutes. Le liquide est repris puis dilué dans l'eau tamponnée. Les germes sont dénombrés dans ces dilutions par incorporation de 1 millilitre du liquide dans le milieu gélosé (germes totaux) ou par étalement de 1 goutte de liquide à la surface du milieu sélectif. Le second prélèvement est mis en suspension de la même 
manière, dans la même quantité d'eau distillée non tamponnée. Ce liquide est utilisé pour la détermination du $p H$ (appareil à électrode de verre).

\section{Méthode des porte-germes.}

La méthode directe a l'inconvénient d'exiger une très grande quantité de suspension microbienne pour que la concentration des germes pathogènes dans la zône de fumier artificiellement infectée soit assez grande ; cette quantité de liquide risque de dissoudre une partie du superphosphate et de fausser les résultats.

La méthode des porte-germes n'a pas ces inconvénients.

Les porte-germes sont constitués de compresses de gaze stérile de $30 \mathrm{~cm}$ x $30 \mathrm{~cm}$, pliées en $10 \mathrm{~cm} \times 7,5 \mathrm{~cm}$. Ces compresses absorbent, à saturation, de 12 à 15 millilitres d'eau. Au début de l'essai, elles reçoivent 10 millilitres de la suspension de staphylocoques, contenant environ $5.10^{9}$ cellules par millilitre.

Dix compresses sont placées dans les deux cases sur la litière et recouvertes d'une mince couche de la même litière. Des compresses témoins, imprégnées d'eau tamponnée à $0,1 \%$, sont disposées à côté des précédentes. La litière est tassée par piétinement. Une compresse est reprise immédiatement; les neuf autres sont reprises à raison de trois par jour les trois jours suivants. Les compresses témoins sont reprises le dernier jour.

Chaque jour, les compresses sont retrouvées à une profondeur un peu plus grande dans la couche de litière, à tel point que, le troisième jour, elles font partie intégrante de la couche superficielle de fumier. Aussitôt prélevées, les compresses sont introduites dans des flacons de 120 centimètres cubes, contenant 10 millilitres de bouillon stérile. Dès le retour au laboratoire, les flacons sont agités sur shaker pendant environ 10 minutes. Le liquide est repris, puis dilué dans l'eau tamponnée. Les staphylocoques et streptocoques sont dénombrés dans ces dilutions par étalement de 1 goutte $(0,05 \mathrm{ml})$ de liquide à la surface des milieux sélectifs.

\section{B. - Recherche sur la peau}

Après un lavage rapide à l'eau, la mamelle de quatre brebis est badigeonnée avec une compresse imprégnée d'une suspension de staphylocoques contenant environ $5,10^{8}$ cellules par millilitre. Les brebis ainsi contaminées sont laissées dans leurs cases respectives.

Un prélèvement est effectué après 24 heures sur une moitié de la mamelle (environ $100 \mathrm{~cm}^{2}$ ) puis après 48 heures sur l'autre moitié. Le troisième jour, l'opération est répétée sur la surface totale. Ce prélèvement est fait à l'aide d'un tampon de coton et de gaze, monté en écouvillon, stérilisé dans un tube contenant 9 millilitres 
de bouillon. Le tampon est frotté soigneusement sur la surface de prélèvement, puis replongé dans le tube. Après agitation, 1 goutte du bouillon est ensemencée sur milieu sélectif.

\section{III. - Résultats et Discussion}

Les résultats sont exprimés dans les tableaux 1, 2, 3, 4 et 5 .

Tous ces résultats concluent dans le même sens : l'épandage de superphosphate sur la litière ne modifie la survie des germes pathogènes ni dans cette litière, ni sur la peau des animaux placés dessus.

TABLEAU I

FLORE TOTALE (GERMES PAR GRAMME DE LITIÈRE) ET pH DE LA LITIËE DE BERGERIE TRAITÉE OU NON PAR LE SUPERPHOSPHATE

\begin{tabular}{|c|c|c|c|c|c|c|}
\hline Litières & \multicolumn{3}{|c|}{ Avec superphosphate } & \multicolumn{3}{|c|}{ Litières Témoins } \\
\hline Echantillons. & 1 & 2 & 3 & 4 & 5 & 6 \\
\hline$p \mathrm{H} \ldots \ldots$ & 7 & 7 & 6.9 & 9 & 9,3 & 9.35 \\
\hline Population . & $4,8 \cdot 10^{8}$ & $9,4 \cdot 10^{7}$ & $8,1 \cdot 10^{7}$ & $9,2.10^{7}$ & $6,3 \cdot 10^{7}$ & $3.10^{7}$ \\
\hline
\end{tabular}

On observe dans les résultats indiqués quelques différences de faible importance dans le nombre des germes retrouvés d'un échantillon à l'autre; il n'y a là rien d'étonnant, étant donné l'extrême

\section{TABLEAU II \\ MÉTHODE DIRECTE}

$p H$ de la litière de bergerie traitée ou non par le superphosphate.

Nombre de Staphylocoques retrouvés après un temps variable après ensemencement de la litière (Nombre de colonies sur Zebovitz modifié ensemencé avec $0.05 \mathrm{ml}$. de la dilution $10^{-3}$ ).

\begin{tabular}{|c|c|c|c|c|c|c|}
\hline \multirow{2}{*}{$\frac{\text { Litières }}{p \text { H. avant l'essai ......... }}$} & \multicolumn{3}{|c|}{ Avec superphosphate } & \multicolumn{3}{|c|}{ Litière Témoin } \\
\hline & & 7.3 & & & 8.7 & \\
\hline$p H$ pendant l'essai ....... & 8.3 & - & 8.25 & 8.9 & - & 8.6 \\
\hline Prélèvement immédiat .... & & 37 & & & 20 & \\
\hline Prélèvement après 24 heures & & 20 & & & 23 & \\
\hline Prélèvement après 48 heures & & 10 & & & 9 & \\
\hline
\end{tabular}




\section{TABLEAU III \\ MÉTHODE DES PORTE-GERMES}

$p \mathrm{H}$ de la litière de bergerie traitée ou non par de superphosphate.

Nombre de Staphylocoques retrouvés après un temps variable dans les porte-germes incorporés à la litière (nombre de colonies sur Zebovitz-modifié ensemencé avec $0,05 \mathrm{ml}$. de la dilution $10^{-4}$ ).

\begin{tabular}{|c|c|c|c|c|c|c|}
\hline Litières & \multicolumn{3}{|c|}{ Avec superphosphate } & \multicolumn{3}{|c|}{ Litière Témoin } \\
\hline$p \mathrm{H} \quad \ldots \ldots \ldots \ldots \ldots \ldots \ldots$ & \multicolumn{3}{|c|}{7.3} & \multicolumn{3}{|c|}{8.8} \\
\hline Prélèvement après 24 heures & 340 & 320 & 320 & 380 & 260 & 140 \\
\hline Prélèvement après 48 heures & 500 & 240 & 230 & 420 & 320 & 200 \\
\hline Prélèvement après 72 heures & 420 & 416 & 270 & 338 & 72 & 40 \\
\hline $\begin{array}{l}\text { Porte-germes témoins } \\
\text { (non-ensemencés) } \\
\text { après } 72 \text { heures }\end{array}$ & & 0 & & & 0 & \\
\hline
\end{tabular}

\section{TABLEAU IV \\ MÉTHODE DES PORTE-GERMES}

$p$ H de la litière de bergerie traitée ou non par le superphosphate.

Nombre de Streptocoques (No 47-1, Str. agalactiae; No 10-38, Str. uberis) retrouvés après un temps variable dans les porte-germes incorporés à la litière (Nombre de colonies sur milieu TKT ensemencé avec $0,05 \mathrm{ml}$ de la dilution $\left.10^{-4}\right)$.

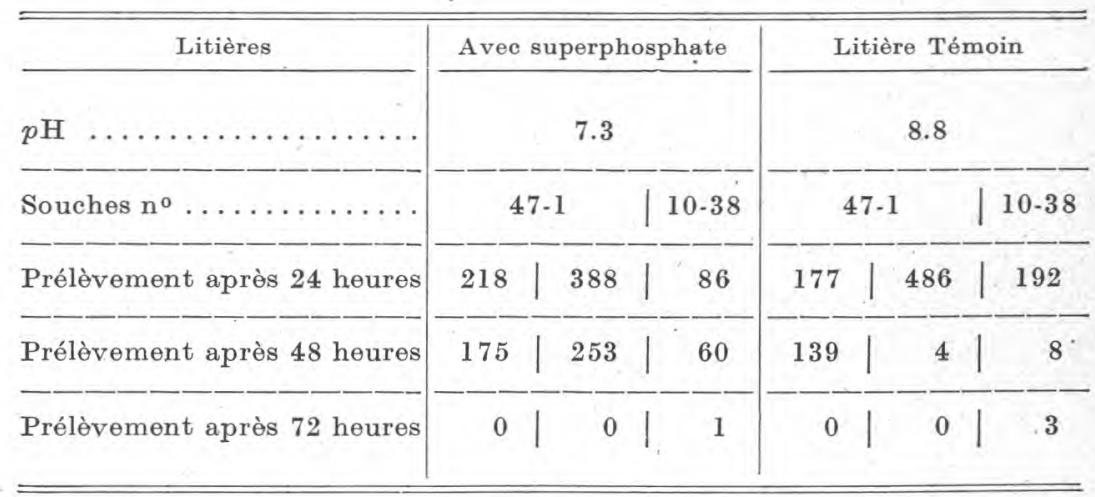

hétérogénéité de la litière. Dans la méthode des porte-germes, certaines compresses retrouvées dans une zone d'excréments sont de véritables parcelles de fumier; d'autres, situées dans une zone 
pailleuse, gardent un aspect presque normal ; les nombres de germes retrouvés dans ces cas extrêmes ne sont pas pour autant très éloignés. Les compresses de la case recevant le superphosphate sont naturellement, selon les hasards de l'épandage, plus ou moins en contact avec ce produit; toutes cependant en sont largement imprégnées ainsi qu'il est facile de le voir à l'abondance du dépôt retrouvé au fond des flacons, au moment de la remise en suspension des germes.

\section{TABLEAU V \\ MÉTHODE DE L'ÉCOUVILLON}

Nombre de Staphylocoques retrouvés après un temps variable sur la peau de la mamelle de brebis après contamination de celle-ci.

Brebis entretenus sur litière avec ou sans superphosphate (Nombre de colonies sur Zebovitz-modifié par 0,05 ml de liquide de prélèvement).

\begin{tabular}{|c|c|c|c|c|}
\hline Litières & Avec $\mathrm{s}$ & sphate & Liti & ooin \\
\hline$\ldots \ldots \ldots \ldots \ldots \ldots$ & 1 & 2 & 1 & 2 \\
\hline Prélèvement après 24 heures & 340 & 100 & 540 & 137 \\
\hline Prélèvement après 48 heures & 4 & 100 & 16 & 30 \\
\hline Prélèvement après 72 heures & 22 & 0 & 92 & 66 \\
\hline
\end{tabular}

Dans la méthode de l'écouvillon, les difficultés de normalisation du prélèvement (délimitation de la surface et vigueur de la friction) font que les différences retrouvées d'un échantillon à l'autre sont assez grandes et ne permettent qu'une estimation assez grossière de la survie des germes.

Quoi qu'il en soit, les différences de survie des germes pathogènes entre les litières témoins et les litières traitées par le superphosphate ne sont jamais en faveur d'une action bactéricide quelconque de ce produit; nous avons au contraire presque constamment trouvé une survie au moins aussi bonne et une population totale plus élevée.

Le $p H$ de la litière de bergerie qui est, dans certains cas, particulièrement élevé $(9,3)$, oscille habituellement autour de 8,8 ; le superphosphate abaisse ce $p H$ aux environs de la neutralité. Même utilisé à des doses très importantes, le superphosphate ne permet jamais d'obtenir des litières à $p H$ acide ; son action bactéricide, réelle en solution aqueuse à $p \mathbf{H}$ acide, est inexistante dans la litière. Le superphosphate n'est done pas un désinfectant des locaux 
et de la litière ; à ce titre, son emploi dans la lutte contre les mammites est sans intérêt.

\section{RÉSUMÉ}

Le superphosphate de chaux en solution aqueuse a un pouvoir bactéricide net sur les microorganismes des mammites (Staphylococcus, Streptococcus, Escherichia). Ce pouvoir bactéricide résulte de l'action en milieu acide de différents ions présents dans la solution. La neutralisation de la solution fait disparaître toute action bactéricide.

L'épandage du superphosphate de chaux sur la litière ne diminue le temps de survie des germes de mammites (Staphylococcus, Str. agalactiae, Str. uberis) ni dans la litière, ni sur la peau de la mamelle des brebis.

\section{BIBLIOGRAPHIE}

[1] G. Lefevre. De l'emploi du Superphosphate sur fumier. Bull. Tech. Inform. 1957, no $121,353$.

[2] S. Metrivier. L'action bactéríostatique du Superphosphate de chaux. Bull. de Docum. 1957, no 21 (Société du Superphosphate).

[3] F. Lagneau. L'action du Superphosphate de chaux et ses répercussions sur l'état sanitaire. Revue de l'Elevage, 1957, no 11.

[4] P. Goret et Ch. Pilet. Activité bactériostatique et bactéricide du Superphosphate de chaux. Rec. Med. Vet., 1957, 133, 1056.

[5] P. Goret et Ch. Pilet. Action bactériostatique et bactéricide du Superphosphate de chaux. C. R. Soc. Biol. 1958, 152, 731.

[6] S. Trocme. Communication personnelle.

[7] G. B. LUDLAM. A selective medium for the isolation of Staphylococcus aureus from heavily contaminated material. Monthly Bull. Ministry of Health, 1949, 8, 15.

[8] G. H. Chapman. Comparison of Ludlam's Medium with Staphylococcus Medium number 110 for the isolation of Staphylococei that clot blood. J. Bact. 1949, 58, 823.

[9] E. Zebovitz, J. B. Evans et C. F. Niven. Tellurite-glycine agar : a selective plating medium for the quantitative detection of coagulasepositive staphylococei. J. Bact. 1955, 70, 686 .

[10] M. Plommet. Le phénomène de Camp. Ses applications au diagnostic, des mammites contagieuses. Milieu T.K.T. Rec. Med. Vet., 1958 134, 285. 